\title{
ARE PERYTONS SIGNATURES OF BALL LIGHTNING?
}

\author{
I. Y. Dodin AND N. J. FisCH \\ Department of Astrophysical Sciences, Princeton University, Princeton, NJ 08543, USA \\ Received 2014 June 11; accepted 2014 August 14; published 2014 September 26
}

\begin{abstract}
The enigmatic downchirped signals, called "perytons," that are detected by radio telescopes in the GHz frequency range may be produced by an atmospheric phenomenon known as ball lightning (BL). If BLs act as nonstationary radio frequency cavities, their characteristic emission frequencies and evolution timescales are consistent with peryton observations, and so are general patterns in which BLs are known to occur. Based on this evidence, testable predictions are made that can confirm or rule out a causal connection between perytons and BLs. In either case, how perytons are searched for in observational data may warrant reconsideration because existing procedures may be discarding events that have the same nature as known perytons.
\end{abstract}

Key words: radiation mechanisms: general - waves

\section{INTRODUCTION}

In the past several years, a number of unusual isolated signals were recorded with the $64 \mathrm{~m}$ Parkes Radio Telescope, Australia, in the frequency range $f \sim 1.2-1.5 \mathrm{GHz}$ (BurkeSpolaor et al. 2011a; Bagchi et al. 2012; Kocz et al. 2012; BurkeSpolaor 2011; Kulkarni et al. 2014). These signals, dubbed "perytons," exhibit a negative chirp $\dot{f} \sim-1 \mathrm{GHz} \mathrm{s}^{-1}$ and last for hundreds of milliseconds. ${ }^{1}$ Also notably, perytons correlate with terrestrial settings such as time of day and weather and are detected in all, or most of, the 13 telescope beams. The common interpretation of the latter is that the signals are picked up by antenna sidelobes and thus must have large spectral flux densities, up to hundreds of kJy (Burke-Spolaor et al. 2011a). An alternative explanation could be (see below) that peryton sources are local and simply have a large enough angular size, namely, $\theta \gtrsim 1^{\circ}$. In either case, the signals are believed to have a terrestrial origin.

Identifying the specific sources of these signals remains an open problem. It is not entirely impossible that perytons are due to a man-made radio frequency (RF) emission. However, this seems unlikely, because perytons cross the band $1.4-1.427 \mathrm{GHz}$, where terrestrial transmitters are legally forbidden to operate (Cohen et al. 2005), and also exhibit an amplitude modulation that, perhaps, excludes hardware failures as their origin (BurkeSpolaor et al. 2011a; Khan 2014). Thus, perytons are more likely to be atmospheric phenomena. However, a specific mechanism through which the Earth's atmosphere produces such RF bursts remains elusive. (For the most recent discussions, see Katz 2014; Khan 2014.) Although perytons do correlate with weather, they are extremely rare compared to weather fluctuations and are not necessarily accompanied by strong wind, rain, or thunderstorms (Bagchi et al. 2012; Burke-Spolaor et al. 2011b). Perytons are therefore not likely to result from common atmospheric phenomena. Rather, they may be emitted by structures, perhaps of decimeter size, that can last for about a second and change their geometry on the same timescale (Katz 2014).

This paper will point out that, although exotic, such atmospheric structures are not unheard of; they are, in fact, widely known as the curious and equally puzzling phenomena called

\footnotetext{
1 The duration of a whole signal must not be confused with the duration of its constituents in individual frequency channels of the telescope, which is typically tens of milliseconds.
}

ball lightning (BLs). Hence, we suggest that perytons are signatures of BLs. Although quantitative data on BLs is scarce, certain parallels between them and perytons are striking, with considerable circumstantial evidence linking these two types of effects. Based on this evidence, testable predictions are made that can confirm or rule out a causal connection between perytons and BLs. Note, however, that our argument does not rely significantly on any specific BL model, which are numerous (Smirnov 1993). In this sense, our paper is not about BLs; rather it builds on some already existing theories of BLs to explain the origin and properties of perytons. We also identify a selection bias in peryton observations and suggest that it is hindering the search for physical mechanisms that could be responsible for generating perytons.

\section{PERYTONS VERSUS BALL LIGHTNINGS}

\subsection{Frequency Range}

BLs are believed by many authors, though not unanimously (Smirnov 1993), to be accompanied by RF activity in just the frequency range where perytons are observed. Indeed, it has been suggested that a BL can serve as a natural electromagnetic cavity (Kapitsa 1955; Watson 1960; Tonks 1960; Silberg 1961; Dawson \& Jones 1969; Jennison 1973; Endean 1976; Muldrew 1990; Zheng 1990; Wessel-Berg 2003). The lowest eigenmode of such a cavity has frequency ${ }^{2}$

$$
f_{c} \sim c / D
$$

where $D$ is the BL diameter and $c$ is the speed of light. Even if the radiation were well-trapped inside the cavity, one can still expect it to radiate somewhat at frequency $f_{c}$. Typically, $D \sim 20 \mathrm{~cm}$ (Smirnov 1993); thus, $f_{c} \sim 1.5 \mathrm{GHz}$, which is close to peryton frequencies.

Measurements of the RF emission naturally generated in thunderstorms also support the theory that BLs represent cavity phenomena. As shown in Kosarev et al. (1968, 1970), the spectral density of this emission (measured at discrete frequencies) increases with frequency at $f \gtrsim 1.0-1.3 \mathrm{GHz}$, in striking

\footnotetext{
2 A geometric factor of order one (Kapitsa 1955) is omitted because $D$ itself varies within almost two orders of magnitude (Smirnov 1993), and perytons may also exist beyond the frequency range within which they are being presently studied.
} 
contrast with the spectral density in the sub-Gigahertz range, which decreases with $f$. It was suggested that this Gigahertz radiation may be associated with BLs (Kosarev et al. 1968, 1970). Note also that the signals in individual frequency channels recorded in those studies are similar to the corresponding signals recorded for perytons.

\subsection{Frequency Chirping}

The only reported quantitative observation of a natural BL (Cen et al. 2014) shows that the BL size can evolve significantly in a fraction of a second. During the quasistationary phase of the $\mathrm{BL}$, this size, in fact, increased at the rate of $\dot{D} / D \sim 0.5 \mathrm{~s}^{-1}$. The value of $D$ itself cannot be inferred directly from the observations in (Cen et al. 2014), which were performed from a large distance $(0.9 \mathrm{~km})$ and, as the authors pointed out, gave only the "apparent" diameter (in the several-meter range) rather than the actual diameter of the BL. However, if one estimates $D$ to be $20 \mathrm{~cm}$, as usual, this leads to $\dot{f}_{\mathrm{BL}} \sim-0.75 \mathrm{GHz} \mathrm{s}^{-1}$. Again, this value is consistent with what is seen for perytons.

Let us now discuss whether the expanding-BL model explains the characteristic shape of $f(t)$ observed for perytons. For this, a brief excursion into the history of peryton studies is needed. Perytons were originally discovered during an archival data survey (Burke-Spolaor et al. 2011a) inspired by the discovery of the so-called Lorimer burst (LB), a similarly shaped chirped Gigahertz signal reported in (Lorimer et al. 2007). As opposed to perytons, the LB was observed in only three beams of the Parkes antenna and thus was identified as a signature of a distant, extraterrestrial event associated with a few-millisecond RF emission. Such an RF signal undergoes dispersive spreading when propagating in space plasma. Specifically, its instantaneous frequency, as detected after time $t$ at a given distance $\ell$ from the source, satisfies (Katz 2014)

$$
\frac{d}{d t}\left[f^{-2}(t)\right]=C(\ell)
$$

where the time-independent $C(\ell)$ is determined by the plasma density integrated along the signal trajectory (also known as the "dispersion measure"). Choosing the value of $C(\ell)$ to fit the observations places the LB origin outside of our galaxy. This motivated the search for other signals that would be similar to the LB, and, through that, perytons were discovered accidentally.

However, we do not know for certain that the observed emission from all perytons follows the relation described by Equation (2). First of all, the very procedure of automatically searching for perytons in archival data introduced a selection bias; e.g., signals corresponding to vanishingly small $C$ and others that were not similar enough to the LB were simply ignored. (One may find this ironic, considering that the similarity between perytons and the LB was later hypothesized to be accidental.) Therefore, Equation (2) may, in fact, reflect properties of the selection algorithm rather than an objective pattern determined by a specific physical effect. Second, even among those perytons that were identified as such, there are some that do not quite satisfy Equation (2). That includes, for example, Peryton 06 in (Burke-Spolaor et al. 2011a) and also some of the more recent observations of peryton-like signals at the Bleien Observatory, Switzerland (Saint-Hilaire et al. 2014). This is particularly notable considering that surveys of Gigahertz bursts cover only a narrow frequency band $(\Delta f / f \lesssim 0.25)$, thus leaving a lot of freedom for fitting. Hence, we may not actually have enough evidence to conclude whether the frequency of perytons, whatever those are, follows a power scaling like Equation (2) or, for that matter, any other universal scaling. ${ }^{3}$ In this sense, the model of a BL as a nonstationary electromagnetic cavity seems to be generally consistent with the peryton frequency chirps that are observed.

\subsection{Observation Patterns}

One of the perytons' puzzling features is that, although they are supposedly atmospheric events, perytons do not correlate significantly with thunderstorms (Bagchi et al. 2012; BurkeSpolaor et al. 2011b). At first sight, this seems to distinguish perytons from BLs, for which such correlations are commonly known (Smirnov 1993). However, the distinction may be caused by another selection bias introduced by observations. BLs are typically detected visually at large viewing angles. Perytons, in contrast, are detected at small viewing angles by RF antennas, which are not even pointed at a storm by default. If perytons are indeed produced by BLs, which are rare events themselves, their generation by distant thunderstorms should be extremely hard to detect this way. Strong storms that occur on site and could produce detectable BLs require stowing radio telescopes ${ }^{4}$ and thus, apparently, have not been monitored.

Hence, we conclude that radiation from BLs observed accidentally by a radio telescope should not be expected to demonstrate significant correlations with thunderstorm proximity. Moreover, lightning strikes may not be the primary cause of BLs in the vicinity of a telescope in the first place. Rather, BLs may spontaneously originate from man-made electromagnetic energy on site, where the presence of large conducting surfaces and, supposedly, powerful electric transformers, makes such events more probable than in natural settings (Smirnov 1993). (The characteristic distance would be $D / \theta \sim 11 \mathrm{~m}$ for $D \sim 20 \mathrm{~cm}$ and $\theta \sim 1^{\circ}$, assuming the Parkes antenna.) If the location of such surfaces relative to the antenna is fixed, that would also explain why the signals appear almost invariably in all of the telescope beams at the same time. Furthermore, since BLs typically move only at moderate speeds, $\sim 1 \mathrm{~m} \mathrm{~s}^{-1}$ (Smirnov 1993), their emission detected by the antenna should be relatively stable on sub-second timescales, and that is precisely what is observed for perytons as well.

Finally, note that, while BLs may not be directly related to thunderstorms, weather may still affect the environment properties; thus, some correlations could be anticipated between weather conditions and the occurrence of even those BLs that do not result from lightning strikes. Although the underlying physics is not understood yet, BLs are typically observed around midday and during foul weather (Smirnov 1993). That is exactly how perytons appear as well, to the extent that their known statistics (Bagchi et al. 2012) can be considered representative. (Of course, the arrival times of some perytons are clustered and may not be entirely random (Burke-Spolaor et al. 2011a; Kocz et al. 2012), but so can be those of BLs, especially if they are produced on-site.) Also, note that even under the most favorable conditions, BLs are still rare events, which is another feature that they have in common with perytons.

\section{RF EMISSION MECHANISM}

Let us now discuss whether our model of perytons can explain how the RF energy is produced or confined long enough

\footnotetext{
3 This is also consistent with the study (Katz 2014) that indicates: if perytons were produced by terrestrial basic plasma effects leading to Equation (2), then the values of $C$ would have been very different from those seen in practice.

4 Parkes Radio Telescope Users Guide, http://www.parkes.atnf.csiro.au/ observing/documentation/user_guide/pks_ug.pdf
} 
within the BL cavity. The existing RF models of BLs (Kapitsa 1955; Watson 1960; Tonks 1960; Silberg 1961; Dawson \& Jones 1969; Jennison 1973; Endean 1976; Muldrew 1990; Zheng 1990; Wessel-Berg 2003) are too sketchy to answer this question; thus, it may be premature to speculate on specifics. On the other hand, there is growing experimental and theoretical evidence that most BL energy may be accumulated in a nonRF form, namely, in the form of internal molecular excitations or chemical energy (Paiva et al. 2007; Dikhtyar \& Jerby 2006; Alexeff et al. 2004; Bychkov 2002; Abrahamson \& Dinniss 2000; Brandenburg \& Kline 1998; Zhil'tsov et al. 1995; Golka 1994; Ohtsuki \& Ofuruton 1991). Thus, a hybrid mechanism may be in effect, such that the RF power does not produce a $\mathrm{BL}$, but is generated as a byproduct through a "plasma maser" mechanism akin to that in (Handel \& Leitner 1994). Specifically, this could work as follows.

With the expected temperature of several thousand Kelvin (Cen et al. 2014), the body of a BL acts as a cold plasma for RF oscillations. This means that its dielectric susceptibility exhibits temporal (but not spatial) dispersion determined by the nonzero electron density, $n_{e}$. For waves with a given angular frequency $\omega=2 \pi f$, the corresponding dielectric susceptibility is $\chi \approx-\omega_{p}^{2} /[\omega(\omega+i \nu)]$, where $\omega_{p}=\left(4 \pi n_{e} e^{2} / m_{e}\right)^{1 / 2}$ is the plasma frequency, $e$ and $m_{e}$ are the electron charge and mass, and $v$ is the electron scattering rate. The scattering is mostly due to collisions with neutrals, and thus $v$ can be taken roughly as a constant, say, $v \sim 10^{12} \mathrm{~s}^{-1}$ (Dawson \& Jones 1969).

The effect of collisions is different for different waves (Aleksandrov et al. 1984). Electrostatic oscillations would decay at the rate of $\sim \mathcal{v}$ and thus are impossible in such plasma in the $\mathrm{GHz}$ range. However, electromagnetic oscillations, whose decay rate is $\sim \nu \omega_{p}^{2} / \omega^{2}$, may be possible. Since $v \gg \omega$ in the frequency range of our interest, we can approximate

$$
|\chi| \sim 5 n_{13} f_{\mathrm{GHz}}^{-1} \sim n_{13} .
$$

Here, $f_{\mathrm{GHz}}$ is the frequency in $\mathrm{GHz}$, and $n_{13}$ is the electron density measured in units of $10^{13} \mathrm{~cm}^{-3}$. It is feasible that the initial electron density is in the range $n_{13} \sim 1$ and is larger at the periphery, as would occur, e.g., in the case of a blast wave. Then, an RF cavity is formed for electromagnetic oscillations, to which excited molecules can emit a fraction of their energy much like in the well-known hydrogen maser (Major 2007). (We suppose that the emission is not particularly sensitive to the cavity size, assuming that many quantum transitions can contribute; e.g., rotational energy of polymer molecules (Bychkov 2002) can be involved, which naturally have a broad distribution of resonant frequencies.) Initially, the RF energy is only poorly confined in such a cavity and will dissipate rapidly, but there is a feedback mechanism that can improve the confinement, namely, as follows.

Note that a BL is expected to consist of dusty plasma (Meir et al. 2013); therefore, $n_{e}$ can vary significantly through absorption and release of electrons from the dust particle surfaces. RF power is one of the determining factors here. As shown experimentally in (Berndt et al. 2006), application of the RF field can decrease the $n_{e}$ in dusty plasma by many times. The specific nature of this effect, which is being debated (Schweigert \& Alexandrov 2012), is not important for our discussion. What is important, however, is that the effect is local and much stronger than that caused by ponderomotive expulsion (Zheng 1990). Already weak RF oscillations may then be able to substantially steepen the $n_{e}$ profile within the plasmoid. Hence, a well-defined electromagnetic mode can form and serve as a narrow-band transmitter of RF radiation at frequency $f_{c}$ (Equation (1)). On the other hand, as the RF energy confinement improves, $n_{e}$ continues to decrease in the BL core, leading to the increase of $D$ and decrease of $f_{c}$; hence, the transmission will be chirped until the maser is exhausted.

Note that the sketch we presented here is intended only to show how one mechanism might possibly be common to perytons and BLs. What we actually draw attention to at this point is merely that the BL expansion is seen in at least some measurements (Cen et al. 2014); thus, in one way or another, chirping of $\mathrm{BL}$ radiation is anticipated. Also, even though most BLs are seen with approximately constant radii, these observations pertain only to long-living structures that can be seen more easily, and, should those emit in the RF range, the radiation would not be identified as perytons (Section 2.2). In contrast, BLs that deteriorate on the sub-second timescale, which is characteristic of perytons, are less likely to be even be noticed and, when they are noticed, are reported as transient.

\section{DISCUSSION}

Our conjecture that two types of curious observations, perytons and BLs, actually result from one and the same phenomenon leads to two predictions. One, we predict that atmospheric BLs emit chirped $\mathrm{GHz}$ radiation. Two, if perytons are indeed signatures of BLs, then they should also emit optical radiation. Facilities that observe perytons do not monitor these optical emissions, but perhaps they should. Also note that should the prediction of either of these emissions be confirmed, it would not only strongly support our theory that perytons and BLs are coincidentally the same phenomenon, but it would also lead to the following consequences.

First, if perytons are indeed signatures of BLs, then they should have a common physical mechanism. Our proposal of such a mechanism here is only a preliminary sketch and describes one of many possibilities. However, what would definitely follow from the coincidence of perytons and BLs is that mechanisms that are not common to both types of observations could then be ruled out. Second, a confirmation of the coincidence of perytons and BLs would suggest that other unidentified curious RF signals should be reconsidered in light of this coincidence. For instance, the LB may not be an extraterrestrial signal after all, as has been already suggested (Kulkarni et al. 2014). This also applies to the similar "fast radio bursts" (FRBs) reported more recently (Thornton et al. 2013 also see references cited therein). The FRBs, including the LB, may be peryton-like signatures of BLs. We might also understand the so-called Wow! signal, a famous yet still-enigmatic $1.42 \mathrm{GHz}$ burst that was received in 1977 by the Big Ear radio telescope, Ohio, and lasted for $72 \mathrm{~s}$ (Gray 2012). It is not unfeasible that although not chirped, this signal is explainable as RF emission from a BL as well, as large enough BLs are indeed known to last over a minute (Smirnov 1993). Thus, what we suggest here is a connection not only between BLs and perytons, but also, possibly, between these curious observations and other known $\mathrm{GHz}$ signals that remain unidentified.

In summary, the hypothesis is advanced here that two types of curious observations, perytons and BLs, actually result from one and the same phenomenon. Although this connection remains speculative, the circumstantial evidence is significant and leads to testable predictions, as summarized in Table 1 . We also point out that, irrespective of whether BLs and perytons are connected, how perytons are searched for in observational data 
Table 1

Summary of the Parallels between BLs and Perytons

\begin{tabular}{|c|c|c|}
\hline & BLs & Perytons \\
\hline Explanation & Unclear & Unclear \\
\hline Observed patterns & $\begin{array}{l}\text { Midday; } \\
\text { usually at thunderstorms, but not only }\end{array}$ & $\begin{array}{l}\text { Midday; } \\
\text { usually on rainy days, but not only } \\
\text { (detectors off during local storms) }\end{array}$ \\
\hline Frequency range & Predicted in the GHz range & Observations limited to $\sim 1.4 \mathrm{GHz}$ \\
\hline Negative frequency chirp & Consistent with cavity expansion & Observed \\
\hline Chirp rate $\sim-1 \mathrm{GHz} \mathrm{s}^{-1}$ & $\begin{array}{l}\text { Predicted } \\
\text { (based on a single observation (Cen et al. 2014)) }\end{array}$ & Observed \\
\hline Chirping consistent with Equation (2) & Possible & Assumed, but not really demonstrated \\
\hline Duration & From a fraction of a second to a minute & Fraction of a second \\
\hline Origin & Terrestrial & Assumed terrestrial \\
\hline Optical emission & Observed & Predicted \\
\hline $\begin{array}{l}\text { Other curious observations } \\
\text { ("Wow!" signal) }\end{array}$ & $\begin{array}{l}\text { Consistent with larger BL } \\
\text { in terms of duration }\end{array}$ & Similar frequency \\
\hline
\end{tabular}

may warrant reconsideration, for existing procedures may be discarding events that have the same nature as known perytons.

This work was supported by the NNSA SSAA grant No. DE274-FG52-08NA28553, by the U.S. DOE contract No. DE-AC02-09CH11466, and by the U.S. DTRA grant No. HDTRA1-11-1-0037. We are also thankful to the reviewer for useful comments.

\section{REFERENCES}

Abrahamson, J., \& Dinniss, J. 2000, Natur, 403, 519

Aleksandrov, A. F., Bogdankevich, L. S., \& Rukhadze, A. A. 1984, Principles of Plasma Electrodynamics (New York: Springer)

Alexeff, I., Thiyagarajan, M., \& Grace, M. 2004, ITPS, 32, 1378

Bagchi, M., Nieves, A. C., \& McLaughlin, M. 2012, MNRAS, 425, 2501

Berndt, J., Kovačević, E., Selenin, V., Stefanović, I., \& Winter, J. 2006, PSST, 15,18

Brandenburg, J. E., \& Kline, J. F. 1998, ITPS, 26, 145

Burke-Spolaor, S. 2011, PhD thesis, Swinburne Univ.

Burke-Spolaor, S., Bailes, M., Ekers, R., Macquart, J.-P., \& Crawford, F., III 2011a, ApJ, 727, 18

Burke-Spolaor, S., Ekers, R., \& Macquart, J.-P. 2011b, in General Assembly and Scientific Symposium, 2011 XXXth URSI (Piscataway; NJ: IEEE)

Bychkov, V. L. 2002, RSPTA, 360, 37

Cen, J., Yuan, P., \& Xue, S. 2014, PhRvL, 112, 035001

Cohen, J., Spoelstra, T., Ambrosini, R., \& van Driel, W. (ed.) 2005, CRAF Handbook for Radio Astronomy (3rd ed.; Strasbourg, France: European Science Foundation), Sec. 7.1

Dawson, G. A., \& Jones, R. C. 1969, PApGe, 75, 247

Dikhtyar, V., \& Jerby, E. 2006, PhRvL, 96, 045002

Endean, V. G. 1976, Natur, 263, 753
Golka, R. K. G., Jr. 1994, JGR, 99, 10679

Gray, R. H. 2012, The elusive Wow: Searching for Extraterrestrial Intelligence (Chicago, IL: Palmer Square Press)

Handel, P. H., \& Leitner, J.-F. 1994, JGR, 99, 10689

Jennison, R. C. 1973, Natur, 245, 95

Kapitsa, P. L. 1955, DoANT, 101, 245

Katz, J. I. 2014, ApJ, 788, 34

Khan, M. D. 2014, arXiv:1404.5080

Kocz, J., Bailes, M., Barnes, D., Burke-Spolaor, S., \& Levin, L. 2012, MNRAS, 420, 271

Kosarev, E. L., Vaganov, A. B., Zakirov, B. S., et al. 1968, ZhTFi, 38, 1831, (Engl. Transl.)

Kosarev, E. L., Zatsepin, V. G., \& Mitrofanov, A. V. 1970, JGR, 75, 7524

Kulkarni, S. R., Ofek, E. O., Neill, J. D., Zheng, Z., \& Juric, M. 2014, arXiv: 1402.4766

Lorimer, D. R., Bailes, M., McLaughlin, M. A., Narkevic, D. J., \& Crawford, F. 2007, Sci, 318, 777

Major, F. G. 2007, The Quantum Beat: Principles and Applications of Atomic Clocks (2nd ed.; New York: Springer), Chap. 11.

Meir, Y., Jerby, E., Barkay, Z., et al. 2013, Mate, 6, 4011

Muldrew, D. B. 1990, GeoRL, 17, 2277

Ohtsuki, Y. H., \& Ofuruton, H. 1991, Natur, 350, 139

Paiva, G. S., Pavao, A. C., de Vasconcelos, E. A., Mendes, O., Jr., \& da Silva, E. F., Jr. 2007, PhRvL, 98, 048501

Saint-Hilaire, P., Benz, A. O., \& Monstein, C. 2014, arXiv:1402.0664

Schweigert, I. V., \& Alexandrov, A. L. 2012, JPhD, 45, 325201

Silberg, P. A. 1961, JAP, 32, 30

Smirnov, B. M. 1993, PhR, 224, 151

Thornton, D., Stappers, B., Bailes, M., et al. 2013, Sci, 341, 53

Tonks, L. 1960, Natur, 187, 1013

Watson, W. K. R. 1960, Natur, 185, 449

Wessel-Berg, T. 2003, PhyD, 182, 223

Zheng, X.-H. 1990, PhLA, 148, 463

Zhil'tsov, V. A., Manykin, E. A., Petrenko, E. A., et al. 1995, JETP, 81, 1072 